\title{
Awareness of Ovarian Cancer Risk Factors among Women in Malaysia: A Preliminary Study
}

\author{
Soon Lean Keng ${ }^{1 *}$,Syakirah Bainun Abdul Wahab ${ }^{1}$, Lim Bee Chiu ${ }^{2}$, Azlina Yusuf ${ }^{1}$
}

\begin{abstract}
Background: Ovarian cancer is recognized as the fourth leading cancer in Malaysia. However, women do not always seek help in a timely manner and gaps in awareness may influence screening uptake and presentation. The purpose of this study was to determine levels of awareness of ovarian cancer risk factors in female population in Penang, Malaysia. Materials and Methods: A cross-sectional study was conducted in Penang, Malaysia from January until February 2014. Eighty-seven women were selected by convenient sampling. Awareness of risk factors of ovarian cancer was assessed using a self-administered questionnaire. Data were analyzed using statistical package for the social sciences (SPSS) version 20.0 for descriptive statistics and Pearson chi-square test for the association between socio-demographic data and awareness. A p value $\leq \mathbf{0 . 0 5}$ was considered statistically significant. Results: In all, $\mathbf{7 4 . 7 \%}$ of participants answered correctly for the risk factor of increasing age, although 94.3\% were unaware of increased risk of tall women. A majority, 71.3\%, had a low level of awareness of ovarian cancer risk factors. There was a significant association between age and knowledge ( $p=0.047)$. Additionally, there was a significant association between higher education level and level of awareness of ovarian cancer risk factors $(\mathbf{p = 0 . 0 3 9 )}$. Conclusions: This study revealed that awareness of ovarian cancer risk factors among Malaysian women is low. The results show a need for improved public understanding about ovarian cancer risks and provision of important information for health professionals about initiatives needed for future awareness, prevention and screening programs.
\end{abstract}

Keywords: Ovarian cancer - awareness - risk factors - women - Malaysia

Asian Pac J Cancer Prev, 16 (2), 537-540

\section{Introduction}

Ovarian cancer is a silent killer and is a challenging disease to treat as currently there is no effective screening test for the disease. For many patients, ovarian cancer is a devastating diagnosis because it is often diagnosed at a late stage (Goff, 2012; Brandner, 2014). The vast majority of cases of ovarian cancer were diagnosed at stage III ( $71 \%)$ or IV $(31 \%)$ and it is related to poor prognosis and survival (Lockwood-Rayermann et al., 2009; Al-Naggar, Muhamed, Bobryshev and Samiah, 2013). In addition, ovarian cancer accounts for more deaths than any other gynecologic cancer such as cervical cancer (Goff, 2012). Delayed in detection of ovarian cancer is predominantly explained by the vague character of the symptoms such as bloated abdomen, abdominal or pelvic pain and urinary symptoms; in combination with lack of awareness and limited knowledge (Bhurgri et al, 2008; Goff, 2012; Brander, 2014). The causes of ovarian cancer are not yet completely understood. Although family history is the single greatest risk factor, however, it explains only 5\% to $15 \%$ of cases of ovarian cancer (Russo et al., 2009). As with most cancers, women of all ages have a risk of developing ovarian cancer. Nevertheless, the risk of developing ovarian cancer is low in young women and increases as women get older.

According to the Malaysia National Cancer Registry in the year 2007 and Penang Cancer Registry (2010), ovarian cancer is the fourth most common cancer among women in Peninsular Malaysia, making up five per cent of all female cancer cases. Most of these women are diagnosed at an advanced stage, where the cancer has spread and is difficult to treat successfully. Al-Naggar et al (2013) found that knowledge on ovarian cancer was poor among Malaysian working women, thus suggesting an annual ovarian cancer campaign nationwide. Prompt awareness may lead to detection at the earliest possible stage of the disease. It is widely documented that early stage diagnosis is associated with an improved prognosis and better survival (Lockwood-Rayermann et al., 2009, Al-Naggar, Muhamed, Bobryshev and Samiah, 2013).

Until recently, most research on awareness of ovarian cancer risk factor and attitudes towards screening more focusing at developed country such as United Kingdom but there was little study done in Malaysia reporting awareness of ovarian cancer Therefore, the purpose of this study was to assess the awareness of ovarian cancer risk factors among women in Malaysia. 


\section{Materials and Methods}

A cross-sectional, descriptive study of the assessment on awareness of ovarian cancer risk factors among women was conducted at the public market in Penang, Malaysia using a self-administered questionnaire. The study was approved by the Human Research Ethics Committee, Universiti Sains Malaysia [USM/JEPeM/275.4.(2.16)]. For ethical consideration, written consent was obtained from the participants when they consented to participate in the study. In addition, they were assured of anonymity and confidentiality throughout the study.

Recruitment and data collection was done concurrently from January to February 2014. All women above 20 years old with no histological diagnosis of ovarian cancer within the study period were invited to participate in the study. The exclusion criteria were women that been diagnosed with ovarian cancer and major psychiatric illness.

\section{Measures}

The RISK questionnaires used were copyright instruments and permission to use them was obtained from the original author (Fallowfield et al., 2010). The questionnaire consists of three sections: Section A, B and C. Section A is on socio-demographic data (independent variable): age, religion, ethnicity and level of education of women. In Section B, the questions was on familiarity of ovarian cancer was measured by descriptive (percentage). In section $\mathrm{C}$, the questions asked about the awareness of ovarian cancer risk factors. These were categorized into adequate awareness and inadequate awareness by giving one mark for correct answer and no mark for the wrong answer and not sure response. The total correct answers were summed and reported as the overall awareness score. The median score of 5 was used as the cut off for defining high or low levels of awareness; whereas those receiving a score less than or equal to the median were classified as having a low awareness of ovarian cancer risk factors following Fallowfield et al. (2009) study.

\section{Analysis}

Data were analyzed using Statistical Package for Social Sciences (SPSS) version 20. Descriptive statistics was used to summarize socio-demographic characteristics of the women, general awareness on ovarian cancer risk factors, awareness on ovarian cancer risk factors and level of awareness on ovarian cancer. Chi square test was used to check the association between socio-demographic characteristics and awareness on ovarian cancer. A p-value of equal or less than 0.05 was considered significant.

\section{Results}

\section{Participant characteristics}

Of the 87 women surveyed, the Malay form the majority in this study $(62.1 \%, \mathrm{n}=54)$. The majority were aged from 20 to 40 years old (86.2\%) and have higher educational level [college and university $(50.6 \%)$ ]. The majority $(50.6 \%)$ were married. More than three-quarters $(82.8 \%)$ of the women surveyed had a monthly income less than RM2500 (785.546 USD) (Table 1).
General awareness of ovarian cancer risk factor

Table 2 shows the results of general awareness of ovarian cancer risk factors among women in Penang, Malaysia. The majority of participants $(85.1 \%)$ heard about ovarian cancer, while $14.9 \%$ never heard about ovarian cancer before. Same goes to question that ask

Table 1. Socio-demographic Characteristics of Participants $(\mathrm{n}=\mathbf{8 7})$

\begin{tabular}{lcc}
\hline Variables & Frequency & Percent (\%) \\
\hline Age (Years) & & \\
$\quad 20-40$ & 75 & 86.2 \\
$\quad 41-60$ & 12 & 13.8 \\
Ethnicity & & \\
$\quad$ Malay & 54 & 62.1 \\
$\quad$ Non-Malay & 33 & 37.9 \\
Education Level & & \\
$\quad$ Low & 43 & 49.4 \\
$\quad$ High & 44 & 50.6 \\
Marital Status & & \\
$\quad$ Single & 38 & 43.7 \\
$\quad$ Married & 44 & 50.6 \\
$\quad$ Divorced & 2 & 2.3 \\
$\quad$ Widowed & 3 & 3.4 \\
Monthly Income (MYR) & & \\
$\quad \leq 2500$ & 72 & 82.8 \\
$\quad$ 2500 & 15 & 17.2 \\
\hline
\end{tabular}

Table 2. General Awareness on Ovarian Cancer Risk Factors $(\mathbf{n = 8 7})$

\begin{tabular}{|c|c|}
\hline \multirow[t]{2}{*}{ General awareness about OC risk factors } & Frequency $(\%)$ \\
\hline & No \\
\hline
\end{tabular}

Have you ever heard about ovarian cancer?

$74(85.1) \quad 13(14.9)$

Have you ever read about ovarian cancer?

$$
50(57.5) \quad 37(42.5)
$$

Do you have regular check-up with physician?

$$
25 \text { (28.7) } 62(71.3)
$$

Do you have any family history of ovarian cancer?

Do you have any family history of breast cancer?

$14(16.1) \quad 73(83.9)$

Table 3. Awareness on Ovarian Cancer Risk Factors

\begin{tabular}{|c|c|c|}
\hline \multirow{2}{*}{ Risk factors of Ovarian Cance } & \multicolumn{2}{|c|}{ Frequency $(\%)$} \\
\hline & Correct answer & Wrong answer \\
\hline Increasing in age & $65(74.7)$ & $22(25.3)$ \\
\hline $\begin{array}{l}\text { Family history with ovarian } \\
\text { breast cancer or cervical } \\
\text { cancer }\end{array}$ & $56(64.4)$ & $31(35.6)$ \\
\hline Genetic predisposition & $58(66.7)$ & $29(33.3)$ \\
\hline Tall women & $5 \quad(5.7)$ & $82(94.3)$ \\
\hline Has never been pregnant & $16(18.4)$ & $71(81.6)$ \\
\hline
\end{tabular}
$(\mathrm{n}=87)$

Table 4. Level of Awareness on Ovarian cancer Risk Factors $(\mathrm{n}=\mathbf{8 7})$

\begin{tabular}{lc}
\hline Awareness level & Frequency (\%) \\
\hline Low $(0-49 \%)$ & $62(71.3 \%)$ \\
High $(50 \%-100 \%)$ & $25(28.7 \%)$ \\
\hline
\end{tabular}


Table 5. Association Between Socio-demographic Characteristics and Awareness of Participants on Ovarian Cancer Risk Factors (n=87)

\begin{tabular}{|c|c|c|c|c|}
\hline \multirow[t]{2}{*}{ Variables } & \multicolumn{2}{|c|}{ Overall awareness, $\mathrm{n}(\%)$} & \multirow[b]{2}{*}{$\mathrm{X}^{2}$ value $^{\mathrm{a}}$} & \multirow[b]{2}{*}{$\mathrm{p}$ value } \\
\hline & Low & High & & \\
\hline \multicolumn{5}{|l|}{ Age (years)* } \\
\hline $20-40$ & $51(68.0)$ & $24(32.0)$ & 2.829 & 0.047 \\
\hline $41-60$ & $11(91.7)$ & $1(8.3)$ & 1 & \\
\hline \multicolumn{5}{|l|}{ Ethnicity } \\
\hline Malay & $37(68.5)$ & $17(31.5)$ & 0.524 & 0.469 \\
\hline Non-Malay & $25(75.8)$ & $8(24.2)$ & 1 & \\
\hline \multicolumn{5}{|l|}{$\begin{array}{l}\text { Highest } \\
\text { education level }\end{array}$} \\
\hline Lower & $35(81.4)$ & $8(18.6)$ & 4.261 & 0.039 \\
\hline Higher & $27(61.4)$ & $17(38.6)$ & 1 & \\
\hline \multicolumn{5}{|c|}{ Monthly income* } \\
\hline$\leq \mathrm{RM} 2500$ & $53(73.6)$ & $19(26.4)$ & 1.123 & 0.35 \\
\hline$>$ RM2500 & $9(60.0)$ & $6(40.0)$ & 1 & \\
\hline
\end{tabular}

whether participant have ever read about ovarian cancer and the majority answer 'Yes' $(57.7 \%)$. Seventy one percent $(n=62)$ of the participants indicated that they do not have regular check-up with physician. Most of the participants have no family history of ovarian cancer $(93.1 \%)$ or breast cancer $(83.9 \%)$.

\section{Awareness of ovarian cancer risk factors}

Table 3 and 4 shows the scenario of awareness of the women about ovarian cancer risk factors. Most of the participants $(71.3 \%)$ had low awareness level on risk factors for ovarian cancer.

Association between socio-demographic data and awareness of ovarian cancer risk factors

Table 5 shows the association between sociodemographic data and awareness of ovarian cancer risk factors. Age and educational level showed significant association with awareness level of ovarian cancer risk factors $(\mathrm{p} \leq 0.05)$. Younger women and women with higher education showed higher awareness on ovarian cancer risk factors.

\section{Discussion}

The Malay forms the majority in this study (54\%). The explanation is this ethnic race form the largest community in Malaysia. Our study revealed that younger participants, those with a higher level of education had a better awareness of ovarian cancer risk factors, which concurrent with Fallowfield's (2010) and El Rhazi et al (2014) studies. This could suggest that for younger participants, education has an influence on their health condition and greater awareness of ovarian cancer risk factors. The observed positive association between education level and awareness of ovarian cancer risk factor is consistent with results from a previous study by Schulman-Green (2008) that mentioned that more educated women may be better able to access and comprehend information about ovarian cancer, indirectly increased the awareness of ovarian cancer risk factors. Lockwood-Rayerman (2009) also stated in his study that there is an association between level of education and awareness of ovarian cancer risk factors where the higher education levels were more aware than lower educational level.

Our study revealed a low level of awareness of these participants about ovarian cancer risk factors. Nearly three-quarters of them were ignorant about it as revealed by awareness level about ovarian cancer risk factors. This finding is coherent with previously reported finding in Malaysia as well as other countries that awareness on ovarian cancer as well as other types of cancer is low although the population have heard about cancer (Bhurgri et al, 2008; Lockwood-Rayerman's, 2009; Salleh et al, 2011; Al-Naggar et al., 2013; Samat et al., 2014).

Out of all our participants, $74.7 \%, 17.2 \%, 18.4 \%$ respectively could identify increasing age, not been breastfeeding and has never been pregnant as risk factors of ovarian cancer.Out of all our women participants, $74.7 \%$, $17.2 \%, 18.4 \%$ respectively could identify increasing age, not been breastfeeding, has never been pregnant as risk factors of ovarian cancer. Lockwood-Rayerman's (2009) study also reported that the majority of participants were unaware of ovarian cancer risk factors. This means that the Malaysian women had a dearth of awareness of ovarian cancer risk factors as some underestimated the specific risk factors and there are some who have common misconceptions. Even more importantly, there were common misconceptions. For example, our study shows that only $5.7 \%$ of the participants answer correctly for the ovarian cancer risk factor of 'tall women'. As far as risk was concerned, the height of a woman was associated with risk of ovarian cancer (American Association for Cancer Research, 2013). This shows that height still affect the risk of getting ovarian cancer, although the reasons are not clearly stated. (Whiteman, 2013) stated that researchers do not know exactly why tall women is one of the risk factors of ovarian cancer, but it may be related to the fact that tall people grow more, some of the same hormones and other factors that make people grow may also increase the chances that dividing cells become abnormal and turn cancerous. Therefore, future studies should explore the indepth understanding on the issues of awareness on ovarian cancer through qualitative investigation.

In conclusions, the study findings showed that awareness of ovarian cancer risk factors among women is low in Malaysia. Misconception of ovarian cancer risk factors is pronounced. There is a significant different between those from younger and older age women and also those from higher and lower education women. More attention should be given to the older women and lower education women. These findings underline the importance of improving public understanding about ovarian cancer risk factors for future awareness, prevention and screening programmes.

\section{Acknowledgements}

We are very grateful to the participants who participated in this preliminary study. We thank also professor Dr Lesley Fallowfield who was generous in giving her permission to use the RISK questionnaire. We also thank Ms Justine Kilkerr who has been instrumental in this 
study. The study was approved by the Human Research Ethics Committee, Universiti Sains Malaysia [USM/ JEPeM/275.4.(2.16)] and written informed consent obtained from participants.

\section{References}

Al-Naggar RA, Osman MT, Bobryshev YV, Kadir SYA (2013). Ovarian cancer: knowledge of risk factors and symptoms among working Malaysian women. Middle-East J Scientific Res, 14, 549-553.

American Association for Cancer Research (AACR) (2013). Women's height linked to cancer risk, study finds. ScienceDaily. ScienceDaily, 25 July. <www.sciencedaily. com/releases/2013/07/130725141521.htm>.

Fallowfield L, Barrett J, Farewel V, et al (2010). Awareness of ovarian cancer risk factors, beliefs and attitudes towards screening: baseline survey of 21, 715 women participating in the UK collaborative trial of ovarian cancer screening. $\mathrm{Br}$ J Cancer, 103, 454-61.

Lockwood-Rayerman S, Donovan HS, Rambo D, Kuo CW (2009). Women's awareness of ovarian cancer risksand symptoms. Am J Nurs, 109, 36-45.

National Cancer Registry Ministry of Health Malaysia (2007). Malaysia cancer statistics-data and figure peninsular Malaysia, Malaysia.

Penang Cancer Registry. (2010). Report of cancer in penang: 2004-2008.

Russo A, Calo V, Bruno L, et al (2009). Hereditary ovarian cancer. Critical Rev Oncology Hematology, 69, 28-44.

Schulman-Green D, Ercolano E, Dowd M, Schwartz P, McCorkle R. (2008). Quality of life among women after surgery for ovarian cancer. Palliative and Supportive Care, 6, 239-47.

Whiteman H. (2013). Future screening test could detect ovarian cancer earlier. Medical News Today. Retrieved : October 25, 2013 from http://www.medicalnewstoday.com/ articles/265177. 